\title{
Advanced, Fully Implantable Optoelectronic Systems for Battery-free, Multimodal Operation in Neuroscience Research
}

Philipp Gutruf ${ }^{1}$, Vaishnavi Krishnamurthi², Abraham Vázquez-Guardado ${ }^{3,4}$, Zhaoqian $\mathrm{Xie}^{6}$, Anthony Banks $^{5}$, Chun-Ju Su ${ }^{1}$, Yeshou $\mathrm{Xu}^{7}$, Chad R. Haney ${ }^{8}$, Emily A. Waters ${ }^{9}$, Irawati Kandela ${ }^{10}$, Siddharth R Krishnan ${ }^{1}$, Tyler Ray ${ }^{1}$, John P. Leshock ${ }^{11}$, Yonggang Huang ${ }^{6}$, Debashis Chanda ${ }^{3,4}$, John A Rogers ${ }^{12}$

${ }^{1}$ Center for Bio-Integrated Electronics at the Simpson Querrey Institute for BioNanotechnology and the Department of Materials Science and Engineering, Northwestern University, 2145 Sheridan Road, Evanston, IL 60208, USA

${ }^{2}$ Functional Material and Microsystems Research Group and Micro Nano Research Facility, RMIT University, Victoria 3000, Melbourne, Australia

${ }^{3}$ CREOL, The College of Optics and Photonics, University of Central Florida, Orlando, FL 32816, USA

${ }^{4}$ NanoScience Technology Center, University of Central Florida, Orlando, FL 32826, USA

${ }^{5}$ Department of Materials Science and Engineering Frederick Seitz Materials Research Laboratory University of Illinois at Urbana-Champaign, Urbana, IL 61801, USA

${ }^{6}$ Department of Civil and Environmental Engineering, Mechanical Engineering, and Materials Science and Engineering, Northwestern University, Evanston, IL 60208, USA

${ }^{7}$ Key Laboratory of C\&PC Structures of the Ministry of Education, Southeast University, Nanjing 210096, China

Department of Civil and Environmental Engineering, Mechanical Engineering, and Materials Science and Engineering, Northwestern University, Evanston, IL 60208, USA

${ }^{8}$ Center for Advanced Molecular Imaging, Radiology, and Biomedical Engineering, Northwestern University, 2145 Sheridan Road, Evanston, IL 60208-3113, USA

${ }^{9}$ Center for Advanced Molecular Imaging, Northwestern University, 2145 Sheridan Road, Evanston, IL 60208-3113, USA

${ }^{10}$ Developmental Therapeutics Core, Northwestern University, 2145 Sheridan Road, Evanston IL 60208, USA

${ }^{11}$ Department of Biomedical Engineering McCormick School of Engineering and Applied Science, Northwestern University, 2145 Sheridan Road Evanston, IL 60208, USA

${ }^{12}$ Departments of Materials Science and Engineering, Biomedical Engineering, Chemistry, Neurological Surgery, Mechanical Engineering, Electrical Engineering and Computer Science Simpson Querrey Institute \& Feinberg Medical School, Northwestern University, 2145 Sheridan Road, Evanston, IL 60208, USA

\footnotetext{
Abstract

Recently developed classes of ultrasmall, fully implantable devices for optogenetic neuromodulation eliminate physical tethers associated with conventional setups and avoid bulky head-stages and batteries in alternative wireless technologies. The resulting systems enable completely untethered, battery-free operation for high fidelity behavioral studies that eliminate motion constraints and enable experiments in a range of environments and contexts (e.g. social interactions) that would be otherwise difficult or impossible to explore. These devices are, however, purely passive in their electronics design, thereby precluding any
} 
form of active control or programmability; independent operation of multiple devices or of multiple active components in a single device is impossible. This paper introduces a series of important concepts in integrated circuit and antenna design which, taken together, enable low power operation, energy efficient and position and angle independent wireless power harvesting with full user-programmability over individual devices or collections of them, in integrated platforms that have sizes and weights not significantly larger than those of previous, passive systems. The results qualitatively expand options in output stabilization, intensity control and multimodal operation, with broad potential applications in neuroscience research, with specific advances in precise dissection of neural circuit function during unconstrained behavioral studies.

\section{Introduction}

The discovery of microbial opsins and their application in neuroscience research forms the basis for activating targeted neurons by optical means ${ }^{1}$, as an entirely new set of methodologies, known collectively as optogenetics ${ }^{2}$. Broad applications of optogenetics now exist in activation and inhibition of selected behavior centers in the brain and the peripheral nervous system ${ }^{3}$. The delivery of light at appropriate intensities to tissues of interest is critically important to successful optogenetic studies ${ }^{4}$. The simplest and most common methods involve optical fibers ${ }^{5}$ and physically tethered to external light sources, but this type of mechanical and optical interface leads to damage of the surrounding tissue and optical attenuation ${ }^{6}$. The tethers also affect behaviors in uncontrolled ways that can frustrate experimental efforts to decipher mechanisms and functions of the brain. Recently introduced wireless, miniaturized subdermal platforms for delivering light in a battery-free mode of operation provide key advantages by minimizing micro motions and therefore scar formation at the probe interface and by enabling unconstrained, tether-free operation. The latter allows for experimental test setups and protocols without the need for advanced habituation and handling of the animals ${ }^{7}$ while enabling operation in a range of environments and contexts (e.g. social interactions) that would be otherwise difficult or impossible to explore.

Although the latest examples of such tools require only simple electronics, they do not enable precise control over the light intensity and they cannot support multimodal operation or independent control over multiple devices ${ }^{8}$. Instead, operation involves external modulation of the source of radio frequency power, such that the devices themselves require only passive components ${ }^{9}$. Shaping the field intensity to achieve a uniform distribution across a region of interest usually comes at the expense of antenna complexity, where examples include resonant cavities and antenna arrays ${ }^{10}$ which becomes harder with increasing experimental area and layout complexity. The most recent approaches involve magnetic resonant coupling and loop antennas, with the ability achieve field variations of $30-50 \%$ in typical experimental cages, sufficient for many experiments ${ }^{7,10,11}$. Precise regulation and programming output intensity might, however, be necessary in certain demanding cases, such as those that require activation of two distinct opsins ${ }^{12}$ or involve challenges in avoidance of photobleaching ${ }^{13}$. New experimental paradigms that require spatial control over multiple illumination sites with dense optical stimulation in a given subject can also be important 
in studies of cellular level interactions of neurons such as phase precession generation ${ }^{14}$ and have only been shown in restraint or tethered animals. On a macro scale bilateral stimulation that targets distinct brain regions to reveal circuit level interactions is desirable ${ }^{15}$. The technology advances reported here address these requirements directly, without significantly increasing the weight or size, or the basic wireless, batteryfree operation of current simple passive platforms. The use of commercially available electronics and manufacturing schemes offers immediate opportunities for broad distribution to the neuroscience community.

\section{Main Text}

Despite their limitations, tethered optogenetic approaches have the advantage of consistent power output as the animal navigates its environment. This level of signal continuity must be matched by wireless platforms. The strategy for providing precise control over the optical intensity builds on the platform presented in Shin et. al. ${ }^{7}$, which uses magnetic resonant coupling for efficient power transfer with minimal device size, component count and weight. In this work we introduce active electronic circuits without increasing size or volume of the circuitry substantially which offers mechanically robust and overall thin, flexible form factor that is critically important for successful deployment as a subdermal implant. Figure 1 shows the physical and electrical designs of three advanced devices with these essential features, but configured for optically stable optogenetic stimulation/inhibition in small animal subjects such as mice with full control over illumination intensity and spatial location.

Figure 1 (a) presents an exploded view schematic illustration of a device that guarantees that optical output remains at a defined value, when operational, regardless of position or orientation within an experimental enclosure. This device uses an active, feedback-regulated constant voltage supply to an implantable microscale inorganic light emitting diode ( $\mu$-ILED, from a commercial vendor) that serves as the light source for optogenetic modulation. The design features an extendable, articulated implantable needle as previously reported (Fig. S1(a)) for facile positioning in the brain, together with a flexible electronics platform that can be affixed to the surface of the skull in a subdermal fashion. The electrical layout is in Figure 1 (b). The system consists of a purpose designed rectifier made up of specialized, radio frequency (RF) Schottky diodes and a linear dropout regulator (LDO). The power delivered to the implanted $\mu$-ILED, and to a separate, subdermal red indicator $\mu$-ILED, can be adjusted by selecting the current limiting resistors $\mathrm{R} 1$ and R2.

Figure 1 (c) shows the makeup of a multimodal device that is capable of optogenetically modulating neuron activity in four spatially distinct sites with programmable frequency and duty cycle. Here, two separately injectable filaments support two $\mu$-ILEDs each, with detailed dimensions in Fig S1 (b). The electrical design, shown in figure 1 (d), involves minimal component count. The rectification and power regulation components are identical to those described above. An additional microcontroller provides timing and illumination control over the $\mu$-ILEDs. Control over the stimulation parameters follows from a custom 
protocol that relies solely on amplitude modulation of the RF that simultaneously powers the implant, useradjustable in real time.

Finally, Figure 1(e) shows a device that features programmable control over the intensity. The design in figure $1 \mathrm{f}$ involves the same building blocks, including a microcontroller as for the multimodal device, but also integrates a digital to analog converter (DAC) made up of a second order lowpass filter and an operational amplifier in a voltage follower configuration to decouple the $\mu$-ILED load from the lowpass. Here, the added complexity and component count of such a DAC provides non-pulsewidth modulated output, which can lead to false results with the newest generation of fast opsins such as chronos ${ }^{16}$. This DAC can provide output frequencies up to $1.5 \mathrm{kHz}$, well above the response speeds of current opsins, with full control over the optical output intensity. Photographic images of these device types, each balanced on a fingertip, are in figure 1 (g)-(i) and injectable dimensions are shown in figure S1 (c). These images also highlight the thin, flexible and planar form factors that facilitate subdermal implantation.

Critical to the successful operation of all of these devices are designs that efficiently harvest RF power provided via magnetic resonant coupling in common behavioral environments. Figure 2 shows the electrical characterization of the rectifier and the active regulation that enables constant power delivery to the $\mu$ ILEDs. Power delivery to the implant occurs by a single wave rectifier that consists of a matching capacitor for tuning the system to an operational frequency of $13.56 \mathrm{MHz}$ and two Schottky diodes with low forward voltage thresholds for rectifying the $\mathrm{AC}$ signal such that passage through a smoothing capacitor (2.2 $\mu \mathrm{F})$ produces a stable DC voltage. The two-diode setup isolates the RF from the DC side to reduce noise and stabilize the analog and digital circuits. The low internal capacitance of these diodes $(0.2 \mathrm{pF})$ at $13.56 \mathrm{MHz}$ minimizes losses in the rectifier. Additional benefits include isolation of the resonant circuit from the digital electronics. Figure 2 (a) shows the rectification behavior with decreasing Ohmic loads. The peak rectified power occurs at a rectified voltage of $2.5-3.5 \mathrm{~V}$, with steep reductions for voltages below $2.5 \mathrm{~V}$. Operation with a stable rectification voltage of $2.8-3.3 \mathrm{~V}$ allows for high power extraction. The sharp drop-off in rectified power at higher loads can be explained by the Q-factor of the coil, shown in figure s2 (i). Specifically, the Q-factor decreases with increasing load, thereby reducing the coupling efficiency between the primary and secondary antenna.

The available electrical power from the rectifier with a conventional dual loop antenna in standard experimental setups can be measured with a shunt resistor $(5.6 \mathrm{kOhm})$ as a function of input power to a dual loop primary antenna that circumflexes the experimental area (dimensions of the loop are in figure S2 (a) and (b)). The resulting power distribution scales with the input power, where the available power increases with input power while the relative spatial power distribution (uniformity) remains constant, as illustrated in figure S2 (c) for the case of $4 \mathrm{~W}$ of input power and in figure S2 (d) for $8 \mathrm{~W}$ in the case of a standard experimental arena $(30 \times 30 \mathrm{~cm})$. The data also reveal that the size of the enclosure determines the power available at the implant; a mouse home cage, which is about half of the area studied here, shows significantly improved power and uniformity (figure S2 (e) and (f)). The uniformity involves a drop of $\sim 50 \%$ 
in the center of the enclosure, which translates directly into a decrease in optical power, as shown in figure S2 (g), which follows electrical to optical power conversion, shown in figure S2 (h), of an unregulated device as comparison. These results are consistent with uniformity previously reported in literature ${ }^{7}$. Power at the implant also depends on the relative angle to the primary coil, with sharp decreases as this angle increases above a certain threshold (figure S3 (a)). Such behavior can be problematic for certain postures of the animal as it actively explores its environment, particularly problematic for animals that move in $3 \mathrm{~d}$ space such as fish ${ }^{17}$ and birds ${ }^{18}$. Here, an angle independent supply of wireless power becomes a critical aspect of system design for many behavioral paradigms.

To mitigate angle dependent power transmission, we demonstrate a system that combines two resonant magnetic antennas that produce predominantly orthogonal fields, thereby supporting angle independent powering of implants. The antenna system shown in figure 2 (b) consists of an antenna (1) that produces a vertical field and an antenna (2) that produces a horizontal field, as shown in the finite element simulations. The antennas are rapidly multiplexed $(5 \mathrm{kHz})$ to produce a DC power supply, independent of the angular orientation, after passing through the rectifier on the implant.

Resulting measurements of the power distribution of such an active antenna system show correlated field variations in space and angle as shown in figure 2 (c) at a physiologically relevant height of $6 \mathrm{~cm}$ which represents an average height of the cage floor for mice (mice on front paws $3 \mathrm{~cm}$ off ground and on hindlegs $9 \mathrm{~cm}$ off ground). Power levels remain sufficiently high irrespective of angle and position in the cage, verified in figure $S 3(b-d)$ with measurements and corresponding simulation results, to enable operation of devices capable of producing optogenetic stimulus.

The active regulation system summarized in figure 1 (b) eliminates variations in power harvesting. In particular, the direct feedback provided by the electronics guarantees an output fixed to a predetermined voltage, thereby yielding a binary operation, i.e. 'on' to a set value if the RF power is sufficient and 'off' if it is not. The behavior with increasing load is in figure 2 (d). The output voltage is stable until the connected load supersedes the input power capability. The resulting drop in voltage is linear and rapid, which results in the device either performing at the specified power level or not operating at all. The switching performance across a range of commonly used frequencies in optogenetics is in figure S3 (e). Above the dropout voltage of $150 \mathrm{mV}$, at an input voltage of $3 \mathrm{~V}$ with a regulated output voltage of $2.8 \mathrm{~V}$, the efficiency of the regulator is $\sim 85 \%$, independent of load. The regulator also has the additional benefit that the rectifier voltage remains in its optimal range of $2.5-3.5 \mathrm{~V}$ regardless of the load. This feature is especially beneficial for $\mu$-ILEDs with low turn on voltages, as it dramatically increases system efficiencies in comparison to designs that involve directly connected $\mu$-ILEDs. Figure 2 (e) shows the output voltage with an $\mu$-ILED load and the current limiting resistor $\mathrm{R} 1$ set to $3.3 \mathrm{kOhm}$. The results indicate a stable output voltage regardless of orientation and position of the implant, with $0.1 \%$ output variation when powered by a primary antenna input of $8 \mathrm{~W}$ with a multiplexed antenna system $(5 \mathrm{kHz})$. The operation of a device oriented at increasing angles normal to the loop antenna 1 is in figure 2 ( $f$ ) where the device with load ( $\mu$-ILED and R1 3.3k) is 
tilted from 0 to 90 degrees at various heights in the cage. Here we observe stable voltage regardless of position and angle. A visual representation of the experiment is in figure $2(\mathrm{~g})$. This performance is significantly improved over that of a non-multiplexed system figure S3 (f). Measurements that illustrate the expected, stable optical output across the experimental area appear in figure 2(h) here performed with a 0 degrees implant orientation using an automated imaging technique. Optical output power variations realized by setting resistor R1 are shown in figure 2 (i). Operation of two immediately adjacent devices orthogonally oriented is also possible as shown in figure S3 (h) and (i) which has important application for simultaneous operation of multiple devices in a single animal model. A stable electrical output also allows integration of indicator $\mu$-ILEDs that reside subdermally such that their output can be visually observed through the skin/fur. Red (figure 2 (i) inset) LEDs are of interest because rodents have exceptionally low perception in this range of wavelength. Infrared $\mu$-ILEDs represent another option (figure 2 (j)) of interest for automated tracking due to the high contrast against visible wavelengths. In both cases, the intensity can be tuned via the current limiting resistor R2 and can therefore be balanced, as desired, with the current available for the optogenetic $\mu$-ILEDs. In a typical setup, the indicator $\mu$-ILEDs operate at much smaller powers (usually well below $1 \mathrm{~mW}$ ) than the optogenetic $\mu$-ILEDs simply because the red and infrared light can be detected easily through the skin/fur, even at very low intensities.

Another advantage of this technology is the low absorption of power at the operational frequency (13.56 $\mathrm{MHz}$ ). Attenuating tissues and structures found in the body such as optically and acoustically scattering media can prohibit use of other wireless power harvesting schemes based on optics and acoustics, especially in larger animal models. The devices presented here are unaffected by such media and can operate through optically, acoustically and high frequency attenuating media as shown in figure $2(\mathrm{k})$ where an active device operates inside an aqueous foam matrix and through large bodies of water (figure S3 (j)). Multimodal operation, in terms of independent control over the intensity of multiple $\mu$-ILEDs, can also be useful. Such features require wireless communication with the implant, which is challenging due to large power requirements for operating the $\mu$-ILEDs and the limited power availability, especially in large experimental environments. Commercial NFC technologies involve antenna systems specifically designed to support modulation methods for communication, which result in antenna and matching circuits that must have bandwidths of at least $1 \mathrm{MHz}$. This requirement demands low $\mathrm{Q}$ factors for the antennas and, consequently, inefficient power transfer capabilities to receiver antennas that are sufficiently small for implantation in animal models such as mice. A method for one-way communication introduced here circumvents these problems. Figure 3 (a) describes the functionality of the system, where the nonvolatile memory of a microcontroller stores program states and modulation in the primary antenna power serves to trigger these stored states. The program states can include all desired experimental protocols for the implant. A schematic description appears in figure 3 (b).

A modulation scheme that allows the user to switch between program states, as inspired by onewire data connections where the ON time on the bus determines a logical 0 or 1 , is in figure 3 (c). The 
beginning of a data flag is triggered by a specific ON time $\left(\mathrm{t}_{1}\right)$ that initiates communication. Program states are then triggered with subsequent primary power pulses with different $\mathrm{ON}$ times $\left(\mathrm{t}_{2}\right)$. The hardware requirement for this protocol is minimal, as the only facilities needed in the microcontroller are a nonvolatile memory and a set of timers. The result affords a high degree of design freedom, without the use of ASICs or other complex electronics and commercially available schemes such as NFC integrated system on a chip which have major draw backs in comparison to the proposed system (figure S4) that prohibit the operation in such optogenetic implants. The choice of microcontroller its pin count, package, the integrated power management and analog front end can be used for various tasks such as the activation of multiple $\mu$-ILEDs and digital to analog conversion, as examples. Two distinct classes of devices, programmable intensity and multi-shank optogenetic stimulator, demonstrate some of the possibilities. Both devices represent significant advances in wireless, battery-free subdermally implanted devices for optogenetics.

Figure 3 (c) summarizes a device capable of wirelessly controlling the intensity of an $\mu$-ILED, where a sequence of images illustrates a demonstration program that increases the intensity. Figure 3 (d) shows the voltage at the $\mu$-ILEDs where $5 \mathrm{~Hz}$ pulses incrementally increase in voltage, which corresponds to an increase of optical output intensity, a demonstration of this mode is also shown in video SV1. Figure 3 (e) highlights a spatial optical recording of the system in a mouse home cage where the intensity is programmed to switch spatially in a way that divides the optical output in the experimental enclosure. Operation at two distinct heights in the cages, namely at 3 and $6 \mathrm{~cm}$ from the base, demonstrates the stability of the system regardless of spatial position in the experimental environment. This unusual capability might be useful in tuning the stimulation threshold of new opsins in vivo while observing the behavior of the animal.

Figure 3 (f) demonstrates capabilities in individual control of four $\mu$-ILEDs on two bilateral probes designed for independent optogenetic stimulation/inhibition in distinct regions in the brain. This example involves a simple, sequential activation of the $\mu$-ILEDs. Precise timing and stable, rapid turn-on behavior of each light source is described in figure $3(\mathrm{~g})$, where each $\mu$-ILED is set to be activated sequentially with a frequency of $1.25 \mathrm{~Hz}$ and a duty cycle of $10 \%$, demonstrating independent control. Any physiologically relevant frequency, duty cycle or sequence can be programmed. Video SV2 presents various programs at frequencies visible to the naked eye, in various modes, all wirelessly activated.

The communication protocol also allows for individual addressing of multiple devices in one experimental enclosure without the need for additional hardware. Addressing of individual devices can be achieved via dedicated pulse length combinations ( $t_{1}$ and $t_{2}$ figure $3(b)$ ) for each device, complementary program state description or unique device ID. Individual addressing of the devices is demonstrated in video SV3 where two bilateral devices are controlled individually in the same RF field, synchronous operation as well as individual stimulation modes are shown. The addressing speed of this system depends on the LDO settling time and overall buffer capacitance in the system, ultimately limiting the number of device that can 
be controlled simultaneously. Advances in addressing speed are possible via direct sensing lines that bypass the operation voltage regulation.

The power consumption of these active devices with a microcontroller, including peripherals, is summarized in figure $\mathrm{S} 3 \mathrm{~g}$ ) where the programmable intensity device, which represents the device with the most peripherals, operates at various input voltages simulating the rectified voltage in the field. While active electronics present an overhead in power consumption, efficient energy harvesting schemes provide sufficient headroom to power devices. The optical emission profile of a bilateral device emitting at $475 \mathrm{~nm}$ in the brain appears in figure $3(\mathrm{~h})$ where a Monte Carlo simulation in a turbid media, replicating operation in the brain, shows the resulting local irradiance. The results show an exponential decay away from the light source rendering an effective stimulation radius of 200-500 $\mu \mathrm{m}$, depending on opsin efficiency, in the shape of an ellipsoid based on an input power of $10 \mathrm{~mW} / \mathrm{mm}^{2}$. Detailed slice information can be found in figure S5 (a-e). The activation distance can be extended or decreased by modulating the input power. Detailed information on the distance dependence of the irradiance is in figure S5 (f). The resulting irradiance available at the implants exceeds activation threshold of most known opsins and halerodopsins ${ }^{12}$ and are therefore suitable for broad use for optogenetic activation and inhibition.

The procedure for implanting the devices uses a simple stereotactic surgery that involves opening the scalp, drilling of the skull and positioning the probe, a procedure that does not take more time or skill than an implantation of a fiber optic cannula, making this technology exceptionally easy to adapt without extra training. Although the general steps follow those in Shin et $\mathrm{al}^{7}$, the specifics for the bilateral, multi- $\mu$-ILED device appear explicitly in Figure 4 as a visual guide for implantation of such devices. In particular, Figure 4 (a-e)) shows the positioning of the device at the desired region, opening the skull to facilitate access to the brain, lowering the probe into the brain, fixing the device with cyanoacrylate glue or dental cement to restrict movement of the probes in the brain, release of the device from the stereotactic arm and bending the probe and fixing onto the skull with cyanoacrylate glue and, finally, closing the skin via suturing, a welladapted procedure by most neuroscience researchers. Figure 4 (f) shows an animal two weeks after surgery, highlighting minimal scaring and rapid recovery. The weight of the animals increases postoperatively up to 216 days, as evidence of their healthy status, Figure S6. Evaluations of device function after implantation and imaging indicate stable operation over extended periods of time with devices still functional at the time of manuscript submission (6.9 months after implantation), video SV4 shows the animal 2 weeks after surgery with activated device (stimulation frequency $30 \mathrm{~Hz}, 20 \%$ duty-cycle) in a home cage. Materials and encapsulation schemes follow work in Shin et $\mathrm{al}^{7}$ where device operation has been observed for more than 1.5 years. Additional investigations examine the immunoreactive glial responses and lesions to the brain tissue associated with the probes. Studies include animals with probes implanted for over 200 days, as analyzed with immunohistochemistry. Details are in the methods section, with results in figure S7 (a). Scaring to the brain tissue is smaller than that associated with optical fibers and immunoreactive glial responses are similar. ${ }^{7}$ The programmable intensity device involves similar surgical 
procedures, as in figure S8 Furthermore, animals are minimally impaired by the weight of the devices ( 15 $\mathrm{mg}$ for the regulated, $60 \mathrm{mg}$ for the bilateral and $75 \mathrm{mg}$ for the adjustable intensity devices, respectively). The lightweight nature is shown graphically with an image of device floating on an aqueous foam (figure S3 $(k))$.

All of the devices reported here are compatible with advanced imaging technologies. We demonstrate operation of the implant in an MRI, shown in figure 4 (g) and with additional detail in figure S9. Here, we examine implanted animals with a combination of computer tomography (CT) and magnetic resonance imaging (MRI) to examine the spatial location of the implanted electronics and the probe, as well as state of the surrounding tissue. Figure $4(\mathrm{~h})$ shows a combined image that overlays MRI slices with the 3D CT reconstruction, the superposed $3 \mathrm{D} \mathrm{MRI}$ and $\mathrm{CT}$ images are automatically colorized highlighting the structure implant tissue and bone. Such images can also reveal details associated with the positioning and the artifact. The time requirement for the imaging procedure is also minimal, in the order of half an hour per animal, which makes this a valid tool for postoperative animal control.

Studies of the thermal load during chronic operation of the devices are summarized in figure 4 (i) and (j). Here, a bilateral device set to sequential, continuous operation results in a $25 \%$ duty cycle per $\mu$-ILED, in air, and induces maximum changes in temperature that are less than $1{ }^{\circ} \mathrm{C}$. Detailed investigations as a function of duty cycle are in figure $\mathrm{S} 10(\mathrm{a}, \mathrm{b})$, where performed with the implant (regulated device) in air, which has a thermal conductivity that is an order of magnitude lower than brain tissue. This measurement setup amplifies the effects of thermal load at high powers ( $4 \mathrm{~mW}$ electrical power) to elevate the changes in temperature to levels above the noise of the measuring equipment.. Even in this scenario the tip does not heat above $1.2{ }^{\circ} \mathrm{C}$ at biologically relevant duty cycles of $40 \%$ and below. Images from an infrared camera for the case of the bilateral device reveal increases in temperature that are well below 1 degree, just above the detection threshold of the camera at the locations of the $\mu$-ILEDs. A slight increase in temperature appears at the regulator $\left(\sim 1^{\circ} \mathrm{C}\right)$, a part of the device that is located outside of the skull. Tests in realistic environments, mimicked on the bench with hydrogel (figure $S 10$ (c)), also indicate no significant increases in temperature at the $\mu$-ILED sites and only slight amounts $\left(\sim 1^{\circ} \mathrm{C}\right)$ at the regulator. These data suggest safe operation in animals indefinitely, consistent with previously reported conclusions for passive devices ${ }^{7,19,20}$.

Finite element analysis provides further insights into the distributions of temperature that result from operation of the $\mu$-ILEDs. For these calculations, the $\mu$-ILEDs act as heaters operating at thermal powers that correspond, via the efficiencies of the devices, to specified irradiance levels determined by Monte Carlo simulations. The modeling focuses on the bilateral multi $\mu$-ILED device (layouts in figure $S 1$ (b) and S11 (a)) because this platform presents the most challenging case of thermal load due to its use of four $\mu$-ILEDs in close spatial proximity. The results (figure S11) reveal that for operating conditions typical of those used in optogenetic stimulation, the maximum change in temperature of the brain tissue remains below $0.4{ }^{\circ} \mathrm{C}$. Specifically, with an activation pattern similar to that in figure $3(\mathrm{~g})$ and a thermal power of $4 \mathrm{~mW}$ per $\mu$ - 
ILED, which corresponds to an irradiance $\left(15.15 \mathrm{~mW} / \mathrm{mm}^{2}\right)$ sufficient to activate the most common opsins (figure $\mathrm{S} 11(\mathrm{~b})$ ), the maximum change in temperature is $<0.4^{\circ} \mathrm{C}$, a steady state value that is reached after a few seconds of operation.

The models can also be used to explore a broader space of parameters for optogenetic stimulation, as defined in figures $\mathrm{S} 11$ (i) and (j). At $20 \mathrm{~Hz}$ in a sequential pulsed mode (figure S11 (b)) irradiances of almost $40 \mathrm{~mW} / \mathrm{mm}^{2}$ can be achieved (duty cycle of $10 \%$ for each $\mu$-ILED and thermal input $9 \mathrm{~mW}$ corresponding to $9.9 \mathrm{~mW}$ electrical input) while maintaining the maximum increase in temperature of the brain tissue below $0.5^{\circ} \mathrm{C}$. Higher stimulation frequencies are generally reduce the thermal load on the surrounding tissue.

Figure 5 shows in detail results collected from a female mouse implanted with the bilateral multi- $\mu$-ILEDs device described above, while under light anesthesia (isoflurane/oxygen mixture). The animals were placed on a heated bed and rectal temperature as well as breathing frequency was monitored during the imaging sessions. The safety of the implants was tested prior to the animal sessions and an IR camera analysis (figure S12) of the implant and surrounding tissue during extended MRI image sessions was conducted and no noticeable heating or disruption of implant function was observed.

The resulting slice images in sagittal, coronal and axial orientation are in figure 5 (a-c), shown with minimal post processing. The contrast of bone and dense objects for CT and the contrast of tissue for MRI yields complementary information. As expected, the implant causes some imaging artifacts in CT reconstruction and in MRI, largely due to the semiconductor device components, counterintuitively little artefact is observed at the copper traces including the antenna. Specifically, shadowing effects appear in the CT images, especially in the axial slices. The overall image quality, however, is sufficient to locate devices and their position with respect to the skull and other anatomical features. The MRI images show a surprisingly low amount of artifacts, with only slight distortions and loss of contrast around the components. Most the brain tissue is unaffected. Imaging of the power regulated device yields similar results, as in figure S13 and S14. In general, the quality of the CT and MRI images enable useful insights into the location of the implants and the state of bone and tissue in the animal. Future imaging that is conducted in conjunction with optogenetic stimulus is feasible include operation inside an MRI system, as shown in figure 4 (g), S9 and video SV5.

\section{Conclusion}

The results presented here provide a ubiquitous basis for a range of fully implantable, wireless tools for programmable, multimodal optogenetics that can address a wide range of stimulation needs in neuroscience for the realization of new experimental paradigms. The battery-free, magnetic resonant coupled operation allows for indefinite experimentation on animals that are completely free of physical or inertial motion constraints. Specifically, closed-loop feedback over the emission intensity and a powerefficient, one-way communication protocol enable user-defined, real time control over essential operational parameters while maintaining effective capabilities in energy harvesting regardless of spatial and angular 
position relative to the experimental housing to maintain adequate optical power levels for successful optogenetic stimulation even in large experimental environments. These tools will not only facilitate standard optogenetic experimental studies, but they will also create new opportunities in freely moving animals such as bilateral optogenetic stimulation with individual control over neuronal populations, social experiments with individually stimulated subjects and in vivo, rapid characterization of new opsins via precise control over emission intensity.

\section{Methods}

Device fabrication

Pyralux AP8535R served as a substrate for the flex circuit. The top and bottom copper layers (17.5 $\mu \mathrm{m}$ thick) were structured via direct laser ablation (LPKF U4). Hole filling via pulsed direct current electroplating of copper (LPKF Contac S4) defined the electrical connections between the top and bottom layers. Components with commercial packaging were attached via reflow soldering with low temperature solder (IndiumCorp). The $\mu$-ILEDs were mounted with a pick-and-place tool (Finetech Fineplacer pico ma) using defined force and temperature $\left(180^{\circ} \mathrm{C}\right)$ with an anisotropic conductive paste. The devices were encapsulated with coatings of parylene formed by chemical vapor deposition (14 $\mu \mathrm{m})$. Commercially packaged components such as the $\mu$ Controller and operational amplifiers were further encapsulated with PDMS (DOW corning Sylgard 184) to minimize any foreign body response.

\section{Electronic components}

Small outline, packaged off-the-shelf components served as the basis for the circuits. A low power microcontroller (ATtiny 84 (Atmel)) with wide operational voltage capability provides current to the LED`s. The microcontroller firmware is programmed prior to device assembly. A low dropout (150 mV) low quiescent current $(18 \mu \mathrm{A})$ linear regulator with fixed internal output voltage $(2.8 \mathrm{~V})$ manages power to the implants (ON semiconductor NCP161). The operational amplifier for the programmable output voltage device occupies minimal space (WLCSP $1 \times 1.5 \mathrm{~mm}$ ) and consumes minimal power $(10 \mu \mathrm{A})$. Passive components with 0201 and 0402 package size help to minimize the footprint.

Electromagnetic simulations

The finite element method was adopted in the electromagnetic simulations to determine the magnetic field and the distribution of output voltage from the unregulated device in the multiplexed cage. The simulations used the commercial software Ansys HFSS (Ansys HFSS 13) with tetrahedron elements and adaptive meshing. The default adaptive convergence condition, together with a spherical surface $(1000 \mathrm{~mm}$ in radius) as the radiation boundary, ensured computational accuracy.

Surgical Procedure 
The Institutional Animal Care and Use Committee (IACUC) reviewed and approved protocols for Northwestern University's program for the humane care and use of animals and inspects the animal facilities and investigator laboratories. Evaluation of the implanted devices was performed in compliance with Animal Welfare and Northwestern's IACUC regulations under approved IACUC protocol IS00005877 'Evaluation of biocompatible devices in vivo model'. Animal study was conducted in 20-27gram CD1 IGS mice (Crl: CD1(ICR)). The sterilized device was sterotactically implanted into the right striatum of anesthetized mice. Coordinates are referenced from bregma: $0 \mathrm{~mm}$ antero-posterior, $+2.5 \mathrm{~mm}$ mediolateral and +-1 $\mathrm{mm}$ medio-lateral. The animals were checked daily for distress, pain and moribund condition. Optical intensity distribution calculation

Monte Carlo simulations ${ }^{21,22}$ were carried out to simulate the illumination pattern of the bilateral device set in turbid media replicating operational conditions in the brain. The simulation volume consisted in a rectangular grid with 5003 bins with $25 \mu \mathrm{m}$ bin size. 7.85x106 photons were launched from a rectangular source ( $\mu$-ILED footprint $0.22 \times 0.27 \mathrm{~mm} 2$ ) with $120^{\circ}$ full divergence angle. Photon propagation was performed in brain matter with $0.6 \mathrm{~cm}-1$ absorption coefficient ${ }^{23}, 211 \mathrm{~cm}-1$ scattering coefficient and 0.86 anisotropy factor ${ }^{24}$. The spatial distribution of the photon fluence rate $(\phi[\mathrm{mm}-2])$ was extracted and normalized with respect to the illumination irradiance of one individual $\mu$-ILED source $(10 \mathrm{~mW} / \mathrm{mm} 2)$.

Thermal FEA model:

Finite element modeling was implemented with the commercial software ABAQUS (ABAQUS Analysis User's Manual 2010, V6.10) to study the changes in temperature of brain tissue that is in direct contact the $\mu$-ILED equipped probes. Components of the probe in their actual layouts, including all layers of encapsulation and associated topology was simulated. The tissue and $\mu$-ILEDs was modeled by hexahedron elements (DC3D8) the minimal mesh size was less than $1 / 10$ of the thickness $(4 \mu \mathrm{m})$ of the $\mu$ ILEDs and at least 3 mesh elements for the thinnest layers such as the parylene encapsulation, the mesh convergence of the simulation was ensured. The thermal conductivity, heat capacity and mass density used in the simulations was $0.5 \mathrm{~W} \cdot \mathrm{m}-1 \cdot \mathrm{K}-1,3700 \mathrm{~J} \cdot \mathrm{kg}-1 \cdot \mathrm{K}-1$ and $1050 \mathrm{~kg} \cdot \mathrm{m}-3$ for the brain tissue; $130 \mathrm{~W} \cdot \mathrm{m}-1 \cdot \mathrm{K}-$ 1, $490 \mathrm{~J} \cdot \mathrm{kg}-1 \cdot \mathrm{K}-1$ and $6150 \mathrm{~kg} \cdot \mathrm{m}-3$ for the $\mu$-ILEDs; $377 \mathrm{~W} \cdot \mathrm{m}-1 \cdot \mathrm{K}-1,386 \mathrm{~J} \cdot \mathrm{kg}-1 \cdot \mathrm{K}-1$ and $8920 \mathrm{~kg} \cdot \mathrm{m}-3$ for the Cu traces; $0.15 \mathrm{~W} \cdot \mathrm{m}-1 \cdot \mathrm{K}-1,1510 \mathrm{~J} \cdot \mathrm{kg}-1 \cdot \mathrm{K}-1$ and $1000 \mathrm{~kg} \cdot \mathrm{m}-3$ for the PDMS encapsulation; $0.21 \mathrm{~W} \cdot \mathrm{m}-$ $1 \cdot \mathrm{K}-1,2100 \mathrm{~J} \cdot \mathrm{kg}-1 \cdot \mathrm{K}-1$ and $909 \mathrm{~kg} \cdot \mathrm{m}-3$ for the $\mathrm{PI} ; 0.126 \mathrm{~W} \cdot \mathrm{m}-1 \cdot \mathrm{K}-1,837 \mathrm{~J} \cdot \mathrm{kg}-1 \cdot \mathrm{K}-1$ and $1110 \mathrm{~kg} \cdot \mathrm{m}-3$ for the parylene encapsulation.

MicroCT imaging

Respiratory signals were monitored using a digital system developed by Mediso (Mediso-USA, Boston, MA). Images were acquired with a preclinical microPET/CT imaging system, Mediso nanoScan scanner. Data were acquired with "medium "magnification, $33 \mu \mathrm{m}$ focal spot, $1 \times 1$ binning, with 720 projection views over a full circle, with a $300 \mathrm{~ms}$ exposure time. Three images were acquired, using $35 \mathrm{kVp}, 50 \mathrm{kVp}$, and70 
$\mathrm{kVp}$. The projection data was reconstructed with a voxel size of $68 \mu \mathrm{m}$ using filtered (Butterworth filter) back-projection software from Mediso.

MRI imaging

MRI was performed on a 9.4T Bruker Biospec MRI system with a $30 \mathrm{~cm}$ bore, a $12 \mathrm{~cm}$ gradient insert, and an Autopac automated sample positioning system (Bruker Biospin Inc, Billerica, MA). Respiratory signals were monitored using an MR-compatible physiologic monitoring system (SA Instruments, Stonybrook, NY) and a warm water circulating system was used to maintain body temperature. An actively decoupled 4channel phased array receive-only radiofrequency coil designed for mouse brain (Bruker Biospin, Inc, Billerica, MA) was mounted on the bed. This assembly was centered inside a $72 \mathrm{~mm}$ quadrature volume coil in transmit-only mode (Bruker Biospin, Inc, Billerica, MA). Mice were imaged using an accelerated spin echo sequence (Rapid Acquisition with Relaxation Enhancement, RARE) oriented in axial, sagittal, and coronal directions. The following parameters were used: TR/TE $=2000 \mathrm{~ms} / 40 \mathrm{~ms}$, RARE factor $8, \mathrm{MTX}$ $=256 \times 256$, FOV $2 \times 2 \mathrm{~cm}, 11-17$ slices of $0.75-1 \mathrm{~mm}$ thick (as needed for full brain coverage), and 2 signal averages. Fat saturation was disabled as this was found to slightly reduce image artifacts from the implanted devices. Acquisition time was approximately 2 minutes per scan. The reconstructed data was visualized in Amira 6.4 (FEl, Houston, TX). MRI and microCT images were manually registered as the image artifacts caused by the device precluded automatic image registration. A non-local means filter was applied to the CT data in Amira to reduce image artifacts.

Operational tests of the battery free wireless implant where performed in a 7T Bruker PharmaScan system. Immunohistochemistry

Procedures for Immunohistochemistry follow protocols described in Lu et al. 2018. Mice were anesthetized with Isofluorane and intracardially perfused with ice cold PBS and 4\% paraformaldehyde in PBS. Brains were dissected, postfixed for $24 \mathrm{~h}$ at $4{ }^{\circ} \mathrm{C}$, and cryoprotected with a solution of $30 \%$ sucrose in $0.1 \mathrm{M}$ phosphate buffer $(\mathrm{pH} 7.4)$ at $4{ }^{\circ} \mathrm{C}$ for at least $24 \mathrm{~h}$. Brains were then cut into 30- $\mu \mathrm{m}$ sections on a VT1200 $\mathrm{S}$ Vibrating-blade microtome. Thirty-micrometer brain sections were washed two or three times in PBS and blocked in blocking buffer (PBS containing $0.5 \%$ Triton X-100 and $5 \%$ normal goat serum) for $1 \mathrm{~h}$. Sections were then incubated for $\sim 16 \mathrm{~h}$ at $4{ }^{\circ} \mathrm{C}$ in blocking buffer containing guinea pig anti-GFAP (1:500) and rabbit anti-Iba1 (1:300). Following incubation, sections were washed three times in PBS and then incubated for 2 $h$ at room temperature in blocking buffer containing Alexa Fluor 488 goat anti-rabbit IgG $(1: 1,000)$, Alexa Fluor 568 goat anti-guinea pig lgG (1:1,000), and Neurotrace 435/455 Blue Fluorescent Nissl stain (1:100). Following the secondary antibody, sections were washed three times in PBS and then mounted on glass slides with hardset Vectashield (Vector Labs). All sections were imaged on a Leica SP8 confocal microscope. Gain, exposure time, and z-stack size remained constant throughout each experiment. All images were processed with the same settings by using Fiji ImageJ. 


\section{Acknowledgements}

We acknowledge support from the Center for Bio-Integrated Electronics at Northwestern. DTC is supported by Cancer Center Support Grant P30 CA060553 from the National Cancer Institute awarded to the Robert H. Lurie Comprehensive Cancer Center. Z.X. acknowledges the support from National Natural Science Foundation of China (Grant No.11402134). Y.H. acknowledges the support from NSF (Grant Nos. 1400169, 1534120 and 1635443).

\section{References}

1 Boyden, E. S., Zhang, F., Bamberg, E., Nagel, G. \& Deisseroth, K. Millisecond-timescale, genetically targeted optical control of neural activity. Nature neuroscience 8, 1263-1268 (2005).

2 Deisseroth, K. Optogenetics. Nature methods 8, 26-29 (2011).

3 Park, S. I. et al. Soft, stretchable, fully implantable miniaturized optoelectronic systems for wireless optogenetics. Nature biotechnology 33, 1280-1286 (2015).

4 Pisanello, F. et al. Multipoint-emitting optical fibers for spatially addressable in vivo optogenetics. Neuron 82, 1245-1254 (2014).

5 Yizhar, O., Fenno, L. E., Davidson, T. J., Mogri, M. \& Deisseroth, K. Optogenetics in neural systems. Neuron 71, 9-34 (2011).

6 Chen, R., Canales, A. \& Anikeeva, P. Neural recording and modulation technologies. Nature Reviews Materials 2, natrevmats201693 (2017).

7 Shin, G. et al. Flexible Near-Field Wireless Optoelectronics as Subdermal Implants for Broad Applications in Optogenetics. Neuron 93, 509-521. e503 (2017).

8 Montgomery, K. L. et al. Wirelessly powered, fully internal optogenetics for brain, spinal and peripheral circuits in mice. Nature methods 12, 969-974 (2015).

9 Park, S. I. et al. Stretchable multichannel antennas in soft wireless optoelectronic implants for optogenetics. Proceedings of the National Academy of Sciences 113, E8169-E8177 (2016).

10 Ho, J. S. et al. Self-tracking energy transfer for neural stimulation in untethered mice. Physical Review Applied 4, 024001 (2015).

11 Gutruf, P. \& Rogers, J. A. Implantable, wireless device platforms for neuroscience research. Current opinion in neurobiology 50, $42-49$ (2018).

12 Klapoetke, N. C. et al. Independent optical excitation of distinct neural populations. Nature methods 11, 338-346 (2014).

13 Akerboom, J. et al. Genetically encoded calcium indicators for multi-color neural activity imaging and combination with optogenetics. Frontiers in molecular neuroscience 6 (2013).

14 Harvey, C. D., Collman, F., Dombeck, D. A. \& Tank, D. W. Intracellular dynamics of hippocampal place cells during virtual navigation. Nature 461, 941-946 (2009).

15 Tye, K. M. et al. Amygdala circuitry mediating reversible and bidirectional control of anxiety. Nature 471, 358-362 (2011).

16 Hight, A. E. et al. Superior temporal resolution of Chronos versus channelrhodopsin-2 in an optogenetic model of the auditory brainstem implant. Hearing research 322, 235-241 (2015).

17 Gerlai, R. A small fish with a big future: zebrafish in behavioral neuroscience. Reviews in the Neurosciences 22, 3-4 (2011). 
18 Yartsev, M. M. \& Ulanovsky, N. Representation of three-dimensional space in the hippocampus of flying bats. Science 340, 367-372 (2013).

19 Lu, L. et al. Wireless optoelectronic photometers for monitoring neuronal dynamics in the deep brain. Proceedings of the National Academy of Sciences 115, E1374-E1383 (2018).

20 Samineni, V. K. et al. Fully implantable, battery-free wireless optoelectronic devices for spinal optogenetics. Pain 158, 2108-2116 (2017).

21 Wang, L., Jacques, S. L. \& Zheng, L. MCML-Monte Carlo modeling of light transport in multilayered tissues. Computer methods and programs in biomedicine 47, 131-146 (1995).

22 Keijzer, M., Jacques, S. L., Prahl, S. A. \& Welch, A. J. Light distributions in artery tissue: Monte Carlo simulations for finite-diameter laser beams. Lasers in surgery and medicine 9, 148-154 (1989).

23 Yaroslavsky, A. et al. Optical properties of selected native and coagulated human brain tissues in vitro in the visible and near infrared spectral range. Physics in medicine and biology 47, 2059 (2002).

24 Yona, G., Meitav, N., Kahn, I. \& Shoham, S. Realistic numerical and analytical modeling of light scattering in brain tissue for optogenetic applications. eneuro 3, ENEURO. 0059-0015.2015 (2016). 


\section{Figures}
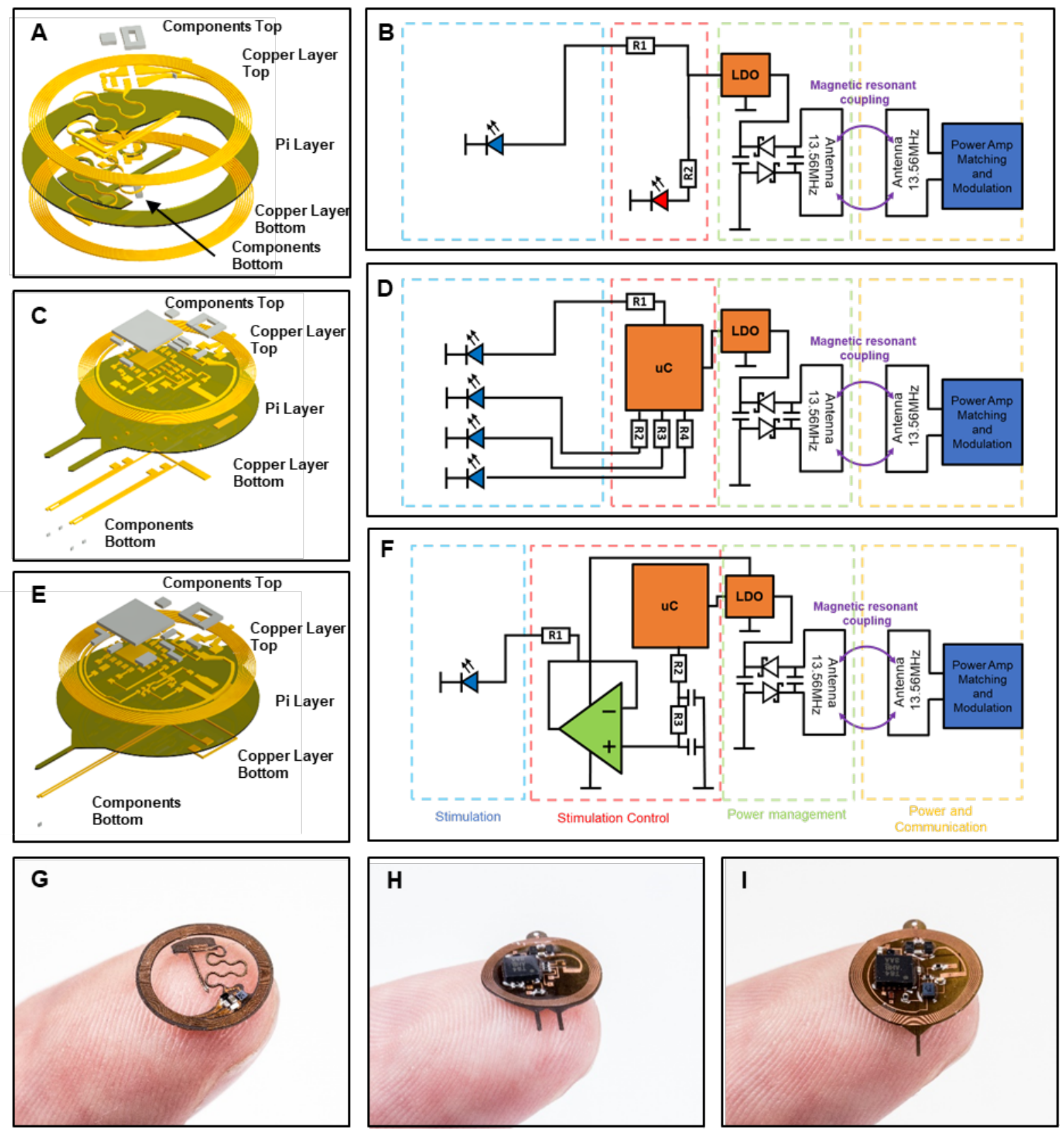

Figure 1 Schematic illustrating $(A)$ layered view of power regulated system with minimal footprint (B) corresponding electrical schematic. (C) Advanced bilateral system with 4 individually and controlled light sources, (D) electrical schematic of the multi $\mu$-ILED device. (E) Layered view of the programmable intensity device. (F) Electrical schematic of the programmable intensity device. (G) Photographic image of the regulated implantable device $(\mathrm{H})$ Photographic image of the programmable multi $\mu$-ILED device. (I) Photographic image of the programmable intensity device 

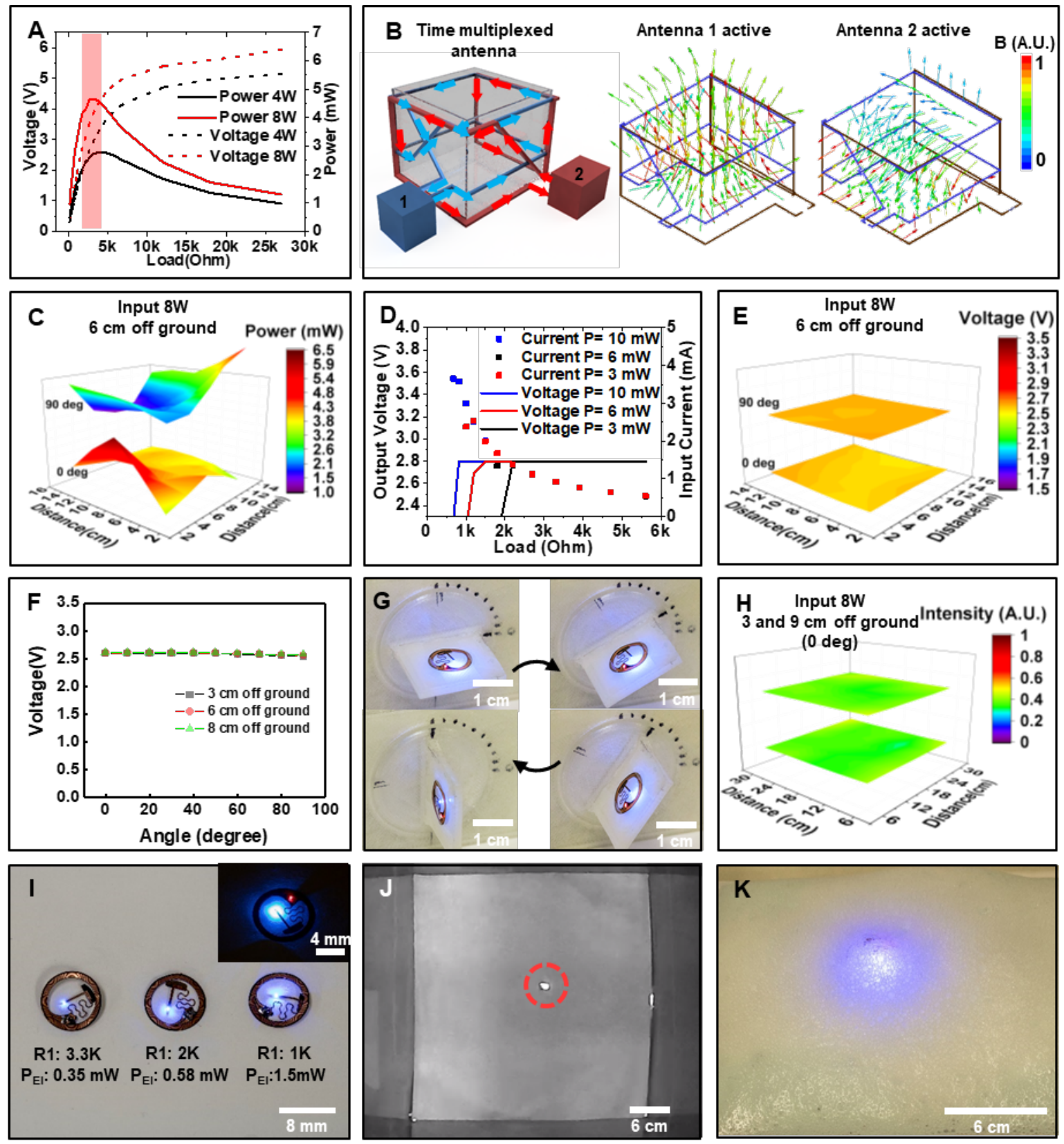

Figure 2 (A) Measurement of rectifier voltage and power with increasing load placed in the center of a field created by a $15 \times 30 \mathrm{~cm}$ cage with $4 \mathrm{~W}$ and $8 \mathrm{~W}$ primary antenna power. (B) Schematic illustration (left) of a time multiplexed antenna system designed to eliminate any angle and position dependence of the operation of the implant. The frames on the right show the results of finite element modeling the resulting magnetic field distribution for each of the two antennas. (C) Rectified power at the implant measured with $8 \mathrm{~W}$ input power applied to a time multiplexed antenna across a cage $(20 \times 20 \mathrm{~cm})$ at an implant height of $6 \mathrm{~cm}$ and at orientations of 0 and 90 degrees with respect to ground. (D) Regulated output voltage and supply current with 3 power levels measured at decreasing load demonstrating turn on behavior of the 
rectifier. (E) Regulated voltage at the implant measured with $8 \mathrm{~W}$ input power in a time multiplexed cage $(20 \times 20 \mathrm{~cm})$ at an implant height of $6 \mathrm{~cm}$ and at orientations of 0 and 90 degree with respect to ground. (F) Measurement of the regulated voltage using an $\mu$-ILED for stimulation and an indicator LED load with 3.3 $\mathrm{kOhm}$ and $5.6 \mathrm{kOhm}$ current limiting resistors, respectively, at a height of 3,6 and $8 \mathrm{~cm}$ above ground with increasing angle, demonstrating stable output throughout angle and space. (G) Photographic images of a regulated implant with increasing angle in a time multiplexed cage using a current limiting resistor on the stimulator $(3.3 \mathrm{kOhm}) \mu$-ILED and indicator $(5.6 \mathrm{kOhm})$ LED. (H) Optical output intensity of a regulated implant at 3 and $9 \mathrm{~cm}$ height in a single primary antenna (power $8 \mathrm{~W}$ in a $30 \times 30 \mathrm{~cm}$ cage). (I) Devices with various power settings for the $\mu$-ILED demonstrating versatility of circuit layout. Inset shows option with red indicator LED. (J) Photographic image with IR sensitive camera in darkness, demonstrating IR indicator light for tracking purposes. (K) Photographic image of a regulated device operating submerged in optically and acoustically attenuating aqueous foam. 

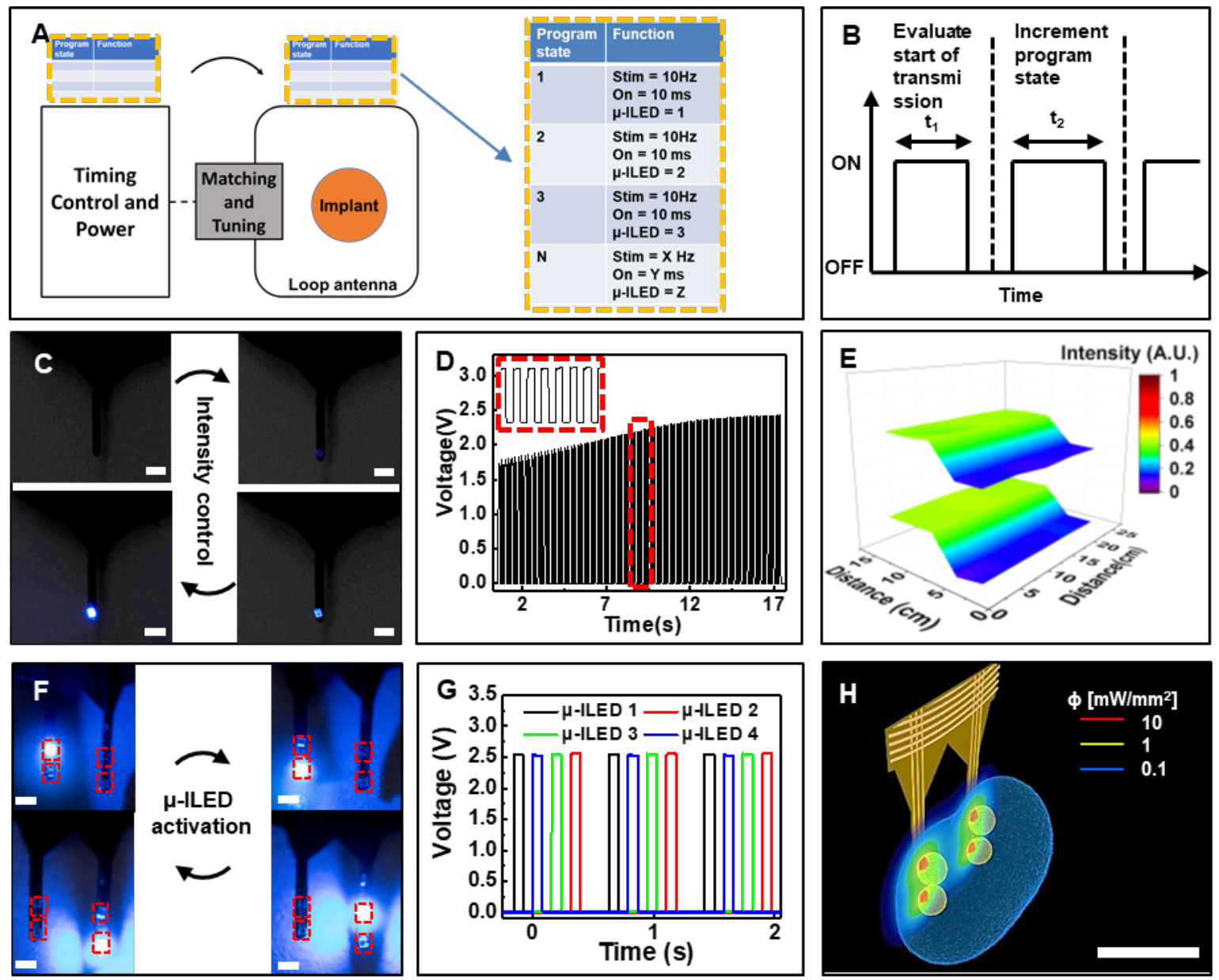

Figure 3 (A) Communication scheme for one way communication to the implant. Inset shows an example of the implants non-volatile memory contend. (B) Timing diagram describing one way connection protocol. (C) Images of the programmable intensity device collected during operation at increasing intensities. (D) Voltage measurement at the $\mu$-ILED during an intensity sweep at a frequency of $5 \mathrm{~Hz}$ where each pulse increases in intensity. (E) Intensity is locally controlled to enable a fixed intensity area inside a test enclosure. The mapping is repeated at 3 and $6 \mathrm{~cm}$ from the cage floor. $(F)$ Demonstration of a bilateral multi $\mu$-ILED device with sequential activation of all stimulating $\mu$-ILEDs. (G) Corresponding voltage traces of all channels displaying stable operation with accurate timing. $(\mathrm{H}) 3 \mathrm{D}$ rendering of Monte Carlo simulations of the operation of a bilateral device at an optical emission of $10 \mathrm{~mW} / \mathrm{mm}^{2}$. 

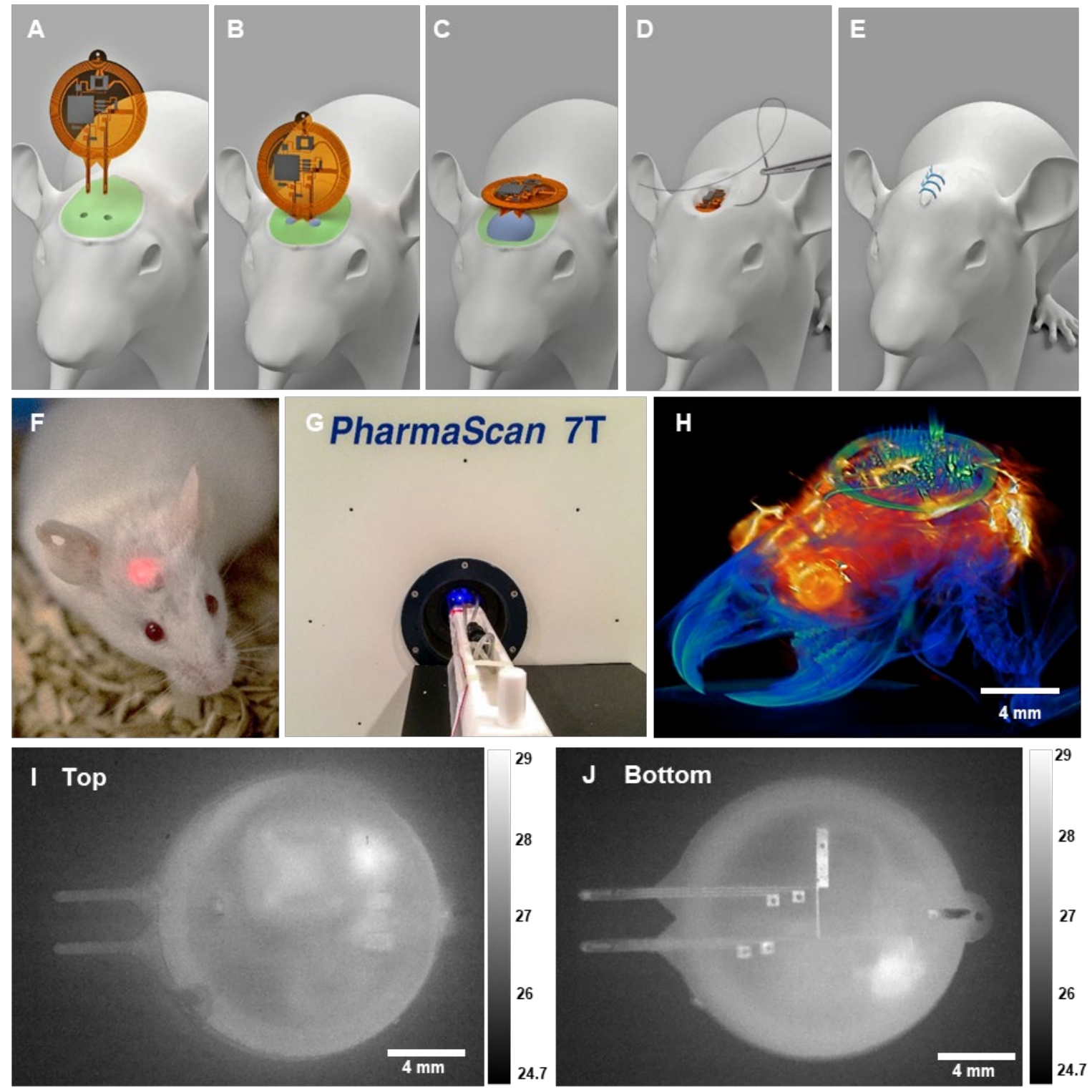

Figure 4 (A-E) Step by step surgical procedure for the implantation of the programmable bilateral multi $\mu$ ILED device. Green colored sections indicate the scull and blue colored sections indicate cyanoacrylate and dental cement glue. (F) Photographic of a mouse 2 weeks after surgery. (G) Implant operating in MRI scanner. $(\mathrm{H})$ Combined image analysis with superposed MRI and CT results superimposed in a $3 d$ rendering of the animal implanted with the programmable bilateral multi $\mu$-ILED device. (I) Thermal image of the top of an operating bilateral device set to $25 \%$ duty cycle on all $4 \mu$-ILEDs (J) Thermal image of the bottom of an operating bilateral device set to $25 \%$ duty cycle on all $4 \mu$-ILEDs. 

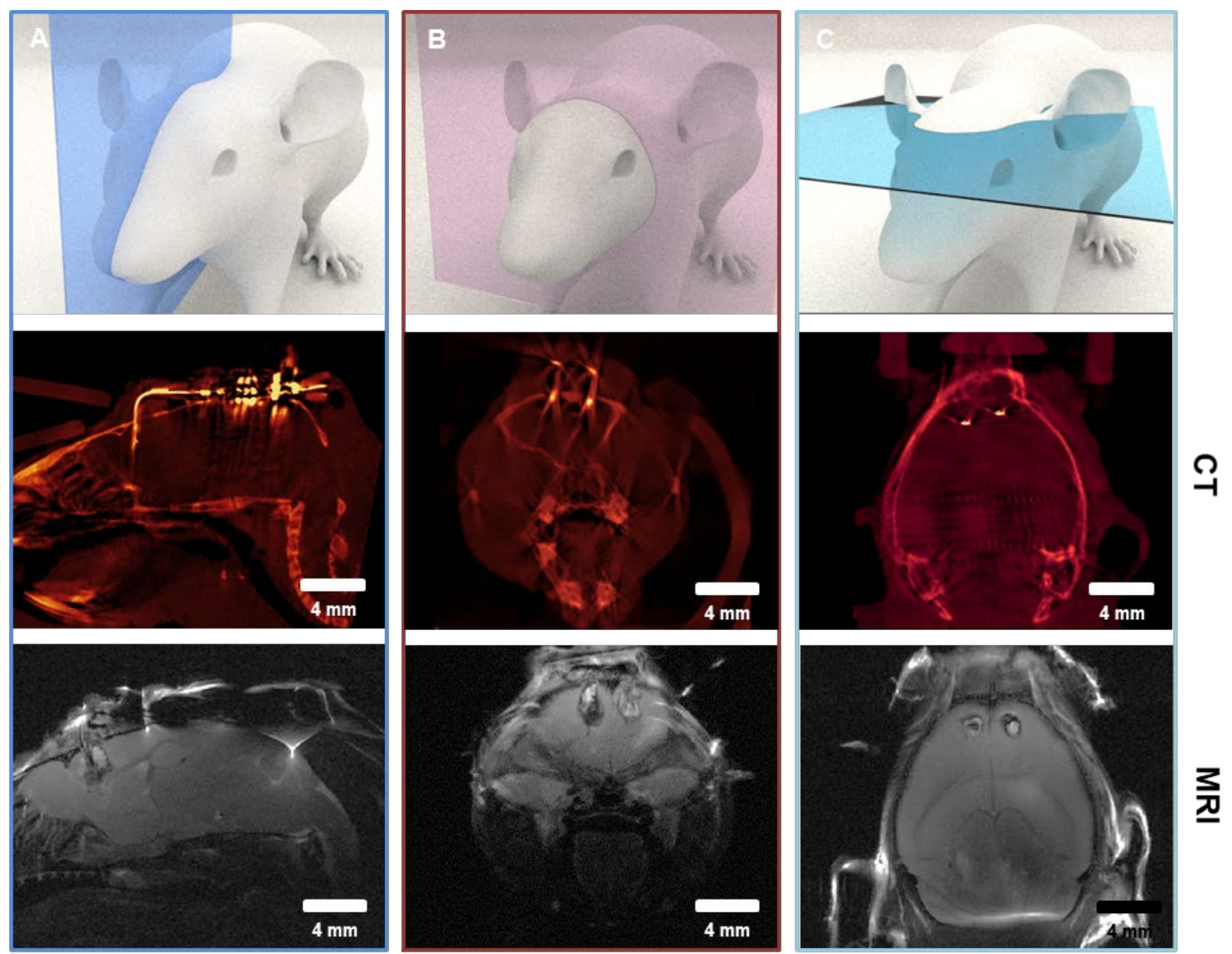

Figure $5 \mathrm{MRI}$ and CT imaging results of the bilateral multi $\mu$-ILED device (A) sagittal orientation, (B) axial and $(\mathrm{C})$ coronal orientation. 